\title{
Tight Control of Systolic Blood Pressure in Spontaneous Intraparenchymal Brain Hemorrhage
}

Mark Krel ${ }^{1}$, James Brazdzionis ${ }^{1}$, James G. Wiginton IV ${ }^{1}$, Dan E. Miulli ${ }^{1}$, Margaret Rose Wacker ${ }^{2}$, Vladimir Cortez ${ }^{3}$

1. Neurosurgery, Riverside University Health System Medical Center, Moreno Valley, USA 2. Neurosurgery, Arrowhead Regional Medical Center, Colton, USA 3. Neurosurgery, Desert Regional Medical Center, Palm Springs, USA

Corresponding author: Mark Krel, m.krel@ruhealth.org

\section{Abstract}

\section{Background}

Tight blood pressure control is critical in neurosurgical patients. Systolic blood pressure (SBP) must be low enough to avoid injury and minimize intraparenchymal hemorrhage (IPH) but high enough to maintain cerebral perfusion. American Heart Association (AHA) guidelines recommend SBP $<140$ in intracerebral hemorrhage. This paper sought to elucidate the effect of early control of SBP on IPH expansion.

\section{Methods}

134 patients with spontaneous IPH between 2011 and 2015 were analyzed utilizing chart review. Initial versus follow-up bleed size, presentation and discharge condition, discharge disposition, and blood pressure control adequacy were analyzed using the generalized linear model.

\section{Results}

Altered mental status was the most common presenting complaint (78\%). Presenting GCS failed to demonstrate a significant main effect. Age, initial IPH volume, presenting SBP, and one-hour SBP significantly affected IPH percent expansion ( $\mathrm{p}=0.002,=0.002,<0.0005$, and $=0.026$ ). Several two-way interactions affected IPH percent change implying synergistic effects of the predictor variables.

\section{Conclusion}

Patients aged 60-70 years had the largest percent IPH expansion followed by patients aged 20-30 years. Initial IPH volume of $65.23-78.26 \mathrm{ml}$ showed the largest expansion. Initial IPH volume of 52.18-65.22 ml demonstrated the least percentage of IPH expansion. One-hour control of SBP to binned groups of 111-121 $\mathrm{mmHg}$ or 121-132 $\mathrm{mmHg}$ portends relative minima in bleed expansion corresponding with AHA

Received 02/23/2019

Review began 04/13/2019 Review ended 05/25/2019 Published 07/23/2019

\section{(c) Copyright 2019}

Krel et al. This is an open access article distributed under the terms of the Creative Commons Attribution License CC-BY 3.0., which permits unrestricted use, distribution, and reproduction in any medium, provided the original author and source are credited. recommendations for IPH patients. This study suggests that this degree of early and aggressive control of SBP is achievable, safe, and may minimize IPH expansion. Future studies are needed to elucidate the role of co-morbidities and to confirm these findings in broader populations.

Categories: Neurosurgery

Keywords: blood pressure, intraparenchymal hemorrhage, systolic

\section{Introduction}

Control of blood pressure is a primary concern in neurosurgical procedures and in the medical management of acute stroke. Arterial blood pressure must be kept low enough to avoid injury and minimize bleeding but high enough to maintain cerebral perfusion in the setting of increased intracranial pressure. Since cerebral perfusion pressure (CPP) is equal to the difference between mean arterial pressure (MAP) and intracerebral pressure (ICP), a balance must be maintained between MAP and ICP to ensure adequate CPP. In the case of increased ICP due to events like hemorrhage or mass effect, the elevated ICP leads to compression of cerebral vasculature. This increased cerebral vascular resistance decreases the capillary flow and can lead to cerebral hypoxia and neuronal cell injury, ultimately resulting in death. Normal autoregulation of cerebral blood flow may be disturbed in settings requiring neurosurgical intervention. Therefore, antihypertensive treatment may need to be tailored depending on the specific clinical situation. Patients experiencing stroke have elevated blood pressure in most cases, regardless of stroke mechanism [1, 2]. In acute ischemic stroke (AIS), there is a linear association between SBP and the event of hemorrhage and increased odds of death [2, 3]. In intracerebral hemorrhagic stroke (ICH), the relationship of increased SBP with death and disability also holds true with an increased in-hospital rate of fatality compared to ischemic stroke, and while the rate of disability may be less than in AIS, it is still of grave concern $[2,4,5]$. 
Hypertension may be directly involved in the secondary expansion of initial hematoma formation [6]. Hematoma expansion (HE) can result in direct mass effect, cerebral edema, and intraventricular hemorrhage leading to hydrocephalus, culminating in increased ICP and decreased CPP [7]. Urgent lowering of blood pressure can minimize hematoma expansion leading to a better outcome $[8,9]$. HE is a suitable predictor of poor outcomes based on the modified Rankin Scale (mRS), with at least a fivefold increased risk of clinical neurological deterioration compared to stable hematoma [10-12]. Early HE has been seen in $26 \%$ of ICH cases at one hour after admission with a further $12 \%$ of patients developing HE by 24 hours [13]. Importantly, neurological deterioration has been shown in $31 \%$ of patients with HE vs $10 \%$ of patients without, and mRS scores and NIHSS are similarly increased when HE occurs. Prompt reduction of SBP is paramount in maximizing patient outcomes.

Therapeutic SBP and MAP goals have been investigated with mixed results that have yet to demonstrate an absolute target SBP value. Based upon the currently available evidence, the American Heart Association (AHA) recommends reducing SBP to $<140 \mathrm{mmHg}$ immediately with SBP $<130 \mathrm{mmHg}$ being a reasonable long-term goal to minimize hematoma expansion, increase in ICP, and risk of re-bleeding [14, 15]. These guidelines state that in IPH, CPP should be maintained between 50 and $70 \mathrm{mmHg}$. Another study suggests $\mathrm{CPP}<80 \mathrm{mmHg}$ may result in less survivability due to hypoxemia as measured on a brain tissue oxygen sensor, not due to hematoma expansion or re-bleed [16].

Small studies have shown favorable outcomes with lower SBP targets. In a study of 211 patients in whom SBP was reduced to $<160 \mathrm{mmHg}$ within one hour of presentation, $\mathrm{HE}>33 \%$ of volume occurred in $17.1 \%$ of patients with neurological deterioration in $8.1 \%$ of patients [17]. Neurological deterioration was described as $\mathrm{a} \geqslant 2$ decrease in GCS or $\geqslant 4$ increase in NIHSS score at $72 \mathrm{hr}$. In this study, $41.2 \%$ of patients had an unfavorable mRS score of $4-6$ at three-months with $1.9 \%$ of patients experiencing mortality by that time. Of note, the average change in the NIHSS score at 72 hours was -2 . Overall, reduction of SBP to $<160 \mathrm{mmHg}$ showed favorable outcomes and was well tolerated.

In a comparison between nicardipine and nimodipine for hypertensive control between 120 and $140 \mathrm{mmHg}$ within one hour in 87 patients, $21.9 \%$ and $18.2 \%$ of patients experienced $\mathrm{HE}>33 \%$ at 24 hours, respectively [18]. Neurological deterioration was seen in $28.3 \%$ of the nicardipine group and $26.8 \%$ of the nimodipine group. A separate study of 88 patients with small-volume ICH and high GCS scores targeted SBP between 100 and $140 \mathrm{mmHg}$ and found only three patients experienced HE, and neurological deterioration was seen in only two patients [19].

While small studies have shown intensive SBP goals to be tolerable and safe, larger trials have described a somewhat limited effectiveness of intensive goals in long-term outcomes. The Intensive Blood Pressure Reduction in Acute Cerebral Hemorrhage Trial (INTERACT) and the follow-up study, INTERACT2, examined the effect of reducing SBP to $<140 \mathrm{mmHg}$ compared with the previously less intensive AHA guideline goal of $<180 \mathrm{mmHg}[20,21]$. INTERACT found $>33 \% \mathrm{HE}$ in $15 \%$ of the intensive group and $23 \%$ of the guideline group, demonstrating a $36 \%$ lower relative risk of HE in the intensive group. Early neurological deterioration was not different between groups (15\% in both) and the 90 -day outcomes of mRS, NIHSS, and serious adverse events were also not different. The follow-up INTERACT2 with 2,794 patients showed no difference in mortality or severe disability at 90 days though ordinal analysis did find modestly improved function in the intensive group. Analysis of the INTERACT2 data has shown that SBP reduction $>20 \mathrm{mmHg}$ and reduction in SBP in the first hour are associated with lower risk of poor outcomes [9]. This data is additionally summarized below in Table 4 in the discussion.

The Antihypertensive Treatment of Acute Cerebral Hemorrhage (ATACH) study assigned patients into treatment groups with goal SBP of 110-139 mmHg, 140-169 mmHg, and 170-200 mmHg to analyze HE based on the degree of SBP reduction [22]. Greater reductions of SBP were associated with less HE and lower mRS scores at three months. The ATACH2 study of 973 patients targeted intensive goal SBP of 110-139 mmHg and less intensive $140-179 \mathrm{mmHg}$ [23]. Death or disability was found in $38.7 \%$ of the intensive group and $37.7 \%$ of the less intensive group, demonstrating no significant difference at three months. Together, the INTERACT and ATACH series of studies have suggested that intensive reduction of SBP to $<140 \mathrm{mmHg}$ does not pose an increased risk to the patient, and while not reducing mortality, it may provide a small increase in functional outcomes.

Early reduction of blood pressure leads to better outcomes in ICH patients, and the target SBP has not adequately been elucidated up to this point. This study examined 134 patients from 2011 through 2015 with ICH and analyzed the reduction in SBP, time to reduction of SBP, HE, and functional outcomes.

\section{Materials And Methods}

One hundred and thirty-four patients seen between 2011 and 2015 at a major county-based hospital for hypertensive intraparenchymal brain hemorrhage were included in this study. Patients who presented for brain hemorrhage but who had underlying pathologic conditions precipitating the hemorrhage such as a mass lesion, vascular lesions, or trauma were excluded from the study. A chart review was performed for each patient who met the inclusion criteria in this study, and the patient's age, ethnicity, presenting complaint, initial IPH size, and blood pressure at one hour were recorded. Size was calculated using 
the $\mathrm{ABC} / 2$ formula for IPH. Then, the size of the IPH on subsequent imaging was recorded. Subsequent imaging was obtained using the standard practice of the neurosurgical center, where-in patients undergo repeat imaging six hours after the initial non-contrast head CT. Lastly, data was collected on the patient's presenting Glasgow Coma Scale Score (GCS) and the patient's GCS at the time of disposition as well as the status of disposition (home, rehab, SNF, other). The data was then analyzed using a generalized linear model with patient age; presenting GCS; initial IPH volume; initial SBP; SBP at one hour; and whether SBP was controlled to < 140, between 140 and 160, or not controlled within one hour. The outcome variable was bleed expansion expressed as a percentage increase or decrease. Wald Chi-Square statistics are reported below.

Analysis was completed using SPSS 23.0 (IBM, Armonk, NY). This study was reviewed and approved for chart review by the institutional review board at Arrowhead Regional Medical Center. Due to the nature of the data collection which utilized a chart review, the institutional review board waived the requirement for formal informed consent.

\section{Results}

In total, 134 patients met the inclusion criteria for this study. The mean age of patients was 59.61 years, with a minimum age of 20 years and a maximum age of 91 years. There were 95 males and 92 females. The most common presenting complaint was altered mental status (78\%) with severe headache as the second-most common complaint (18\%). ICU length of stay ranged from 1 to 34 days with a mean of 6.37 days. Hospital length of stay ranged from 2 to 60 days with a mean of 11 days. Initial presenting GCS ranged from 3 to 15 with a mean of 13.08, while GCS at Day 3 ranged from 8 to 15 with a mean of 13.35 . Initial IPH volume ranged from $0.01 \mathrm{ml}$ to $130.43 \mathrm{ml}$ with a mean of $19.6 \mathrm{ml}$. Follow-up volume ranged from $0.04 \mathrm{ml}$ to 187.44 $\mathrm{ml}$ with a mean of $28.63 \mathrm{ml}$. Percent change ranged from $-79 \%$ to $+1,935 \%$ with average change $103.49 \%$. Initial SBP ranged from $104 \mathrm{mmHg}$ to $260 \mathrm{mmHg}$ with mean $166.07 \mathrm{mmHg}$, and SBP at one hour ranged from $102 \mathrm{mmHg}$ to $209 \mathrm{mmHg}$ with mean $145.79 \mathrm{mmHg}$. See Table 1 for a summary of this data.

\begin{tabular}{|c|c|c|c|c|}
\hline Variable & Minimum & Maximum & Mean & Std Dev \\
\hline Age & 20 & 91 & 59.61 & 17.22 \\
\hline LOS ICU & 1 & 34 & 6.37 & 7.06 \\
\hline LOS hospital & 2 & 60 & 11 & 11.82 \\
\hline GCS initial & 3 & 15 & 13.08 & 3.28 \\
\hline GCS Day 3 & 8 & 15 & 13.35 & 2.46 \\
\hline Initial IPH volume & 0.01 & 130.43 & 19.6 & 26.15 \\
\hline Repeat scan IPH volume & 0.04 & $18 / .44$ & 28.63 & 39.15 \\
\hline$\%$ Change & -79 & 1935 & 103.49 & 268.94 \\
\hline SBP initial & 104 & 260 & 166.07 & 32.03 \\
\hline SBP 1 hr & 102 & 209 & 145.79 & 23.62 \\
\hline
\end{tabular}

TABLE 1: Summary descriptive statistics of variables.

Summary of Abbreviations in Table 1.

Length of stay: LOS

Intensive care unit: ICU

Glasgow Coma Scale: GCS

Intraparenchymal hemorrhage: IPH

Systolic blood pressure: SBP

Standard deviation: Std Dev

Generalized linear model analysis was performed on the data set with patient age; presenting GCS; initial IPH volume; initial SBP; SBP at one hour; and whether SBP was controlled to less than 140, between 140 and 160 , or not controlled to less than 160 within one hour. The outcome variable was hematoma expansion expressed as a percentage increase or decrease. Initial presenting GCS demonstrated no significant main 


\section{Cureus}

effect on percent change in IPH size, $\chi 2(6)=4.958, \mathrm{p}=0.550$ (Figure 1). Patient age demonstrated a significant main effect on percent change in IPH size, $\chi 2(5)=18.830, p=0.002$ (Figure 2). Initial IPH volume demonstrated a significant main effect on percent change in IPH size, $\chi 2$ (3) $=14.472, \mathrm{p}=0.002$ (Figure 3). Presenting SBP demonstrated a significant main effect on percent change in IPH size, $\chi 2(10)=34.230, p<$ 0.0005 (Figure 4). SBP at one hour of presentation demonstrated a significant main effect on IPH size, $\chi 2$ (7) $=15.931, \mathrm{p}=0.026$ (Figure 5, Table 2). Notably, if SBP control was binned by level (i.e., analyzed by three groups where Group 1 was patients whose SBP was controlled to $<140 \mathrm{mmHg}$, Group 2 was patients whose SBP was controlled to $<160 \mathrm{mmHg}$ but not $<140 \mathrm{mmHg}$, and Group 3 was comprised of the remaining patients whose SBPs were not successfully controlled), no significant main effect was discovered, $\chi 2(2)=$ 5.063, $\mathrm{p}=0.080$ (Figure 6). Table 3 summarizes the factors that affected IPH expansion.

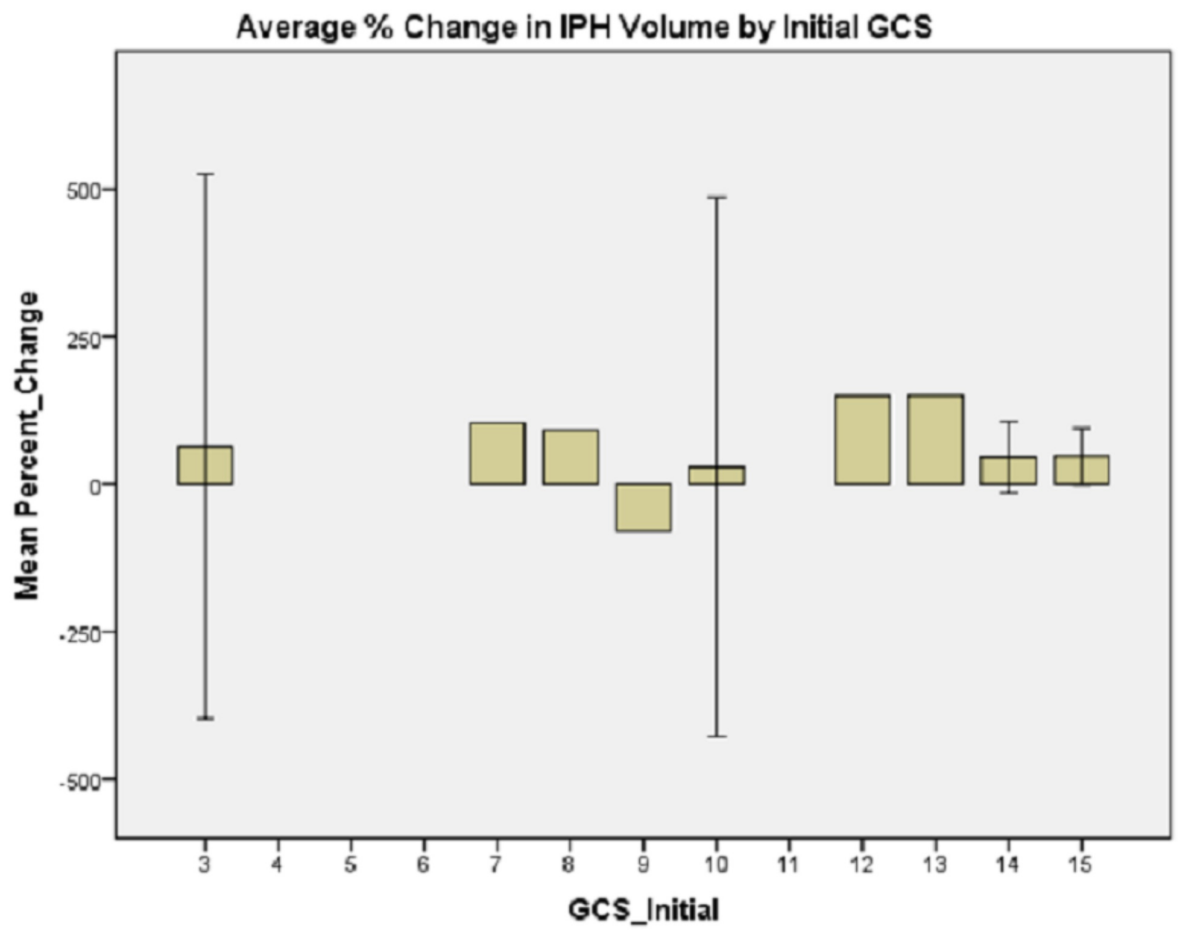

FIGURE 1: Average \% change in IPH volume by initial GCS.

While patients who presented with GCS 10 had, in absolute value, that smallest change in IPH volume, the variance was again quite high and patients whose initial GCS was nine seemed to have an overall decrease in IPH size on follow-up imaging.

Intraparenchymal hemorrhage: IPH

Glasgow Coma Scale: GCS 


\section{Cureus}

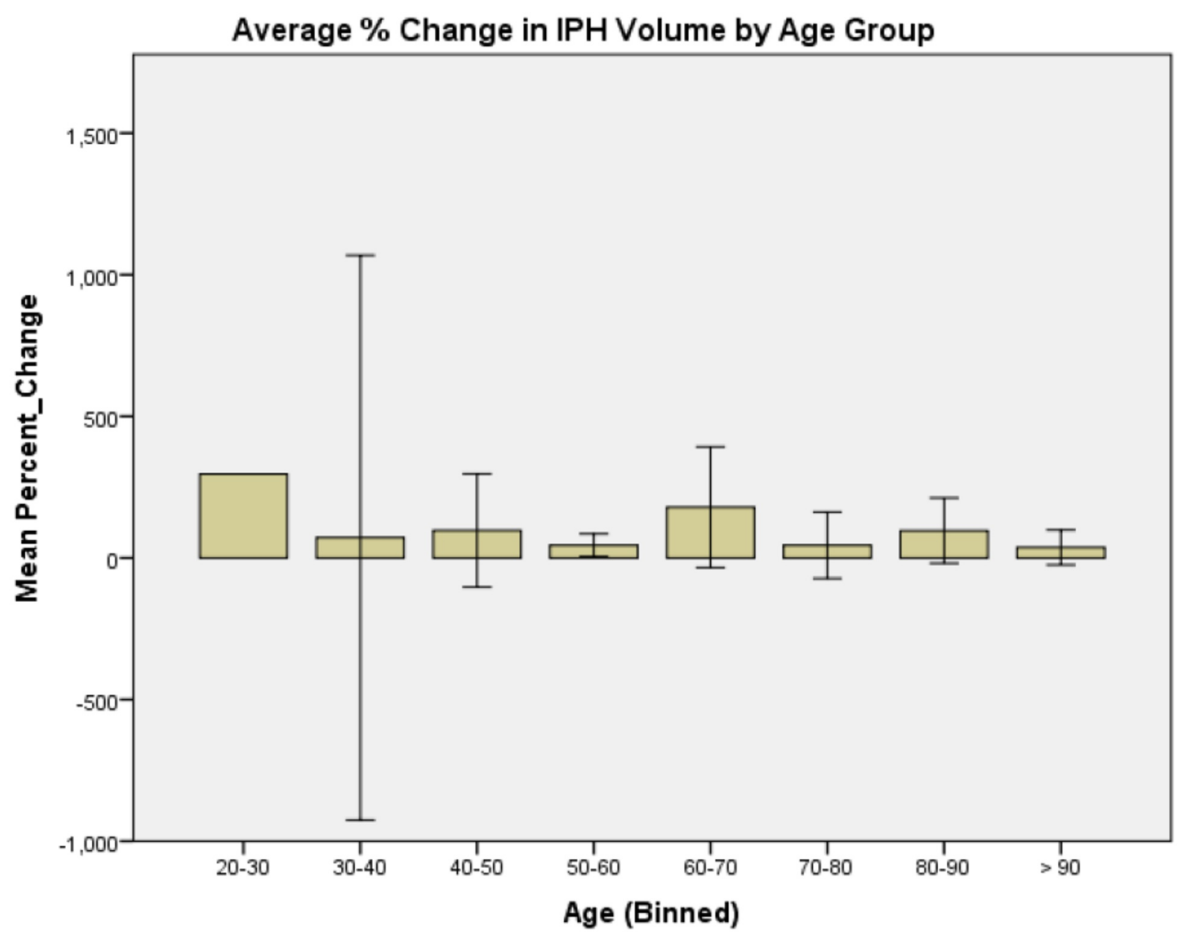

FIGURE 2: Average \% change in IPH volume by age group.

Patients in the 60-70 years age group tended to have the largest IPH expansion followed by the 20-30 years age group.

Intraparenchymal hemorrhage: IPH

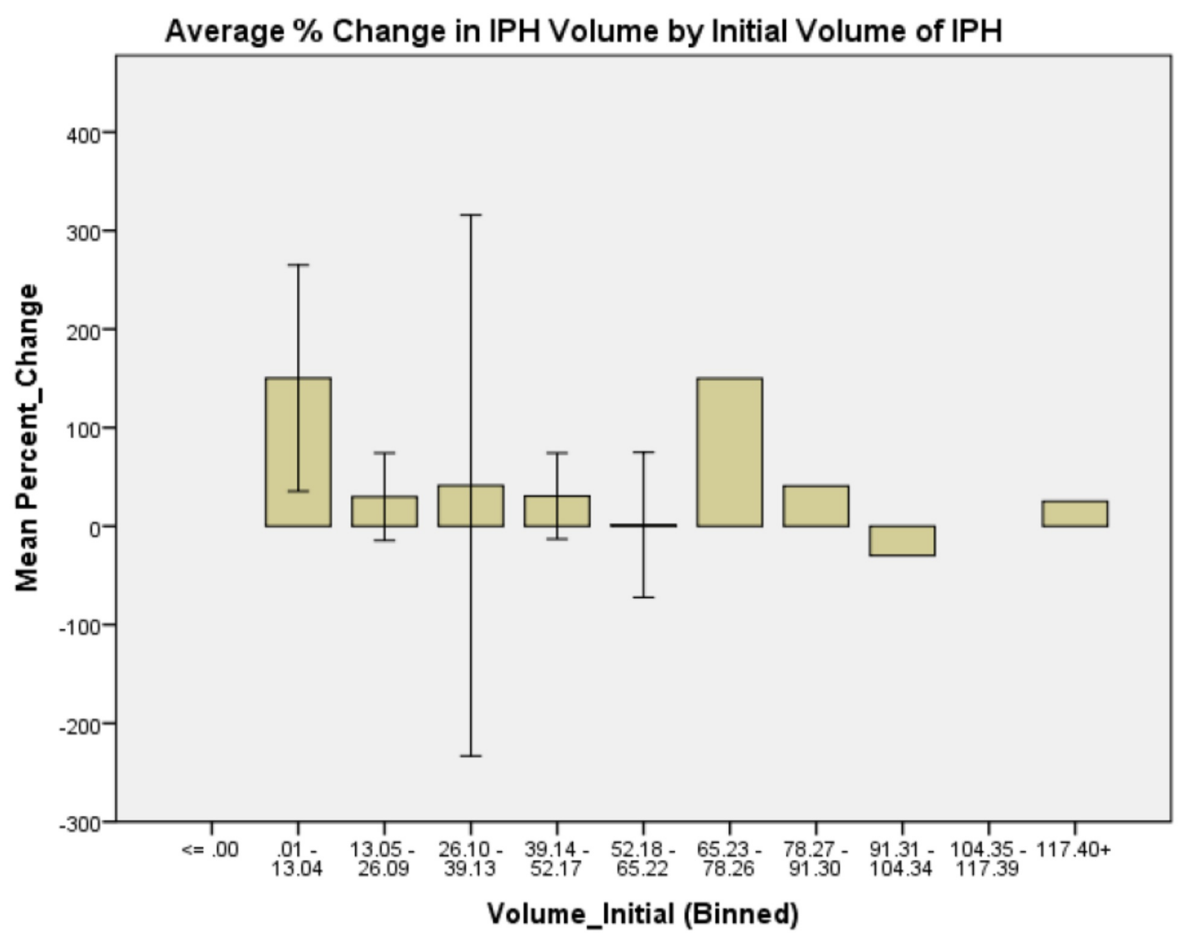

FIGURE 3: Average \% change in IPH volume by initial volume of IPH.

Again, the data is roughly normally distributed with peak expansion occurring in the group of patients who presented with initial IPH volume of 65.23 to $78.26 \mathrm{ml}$. There is also a second maximum in patients who presented with initial IPH volume of 0.01 to $13.04 \mathrm{ml}$. 


\section{Cureus}

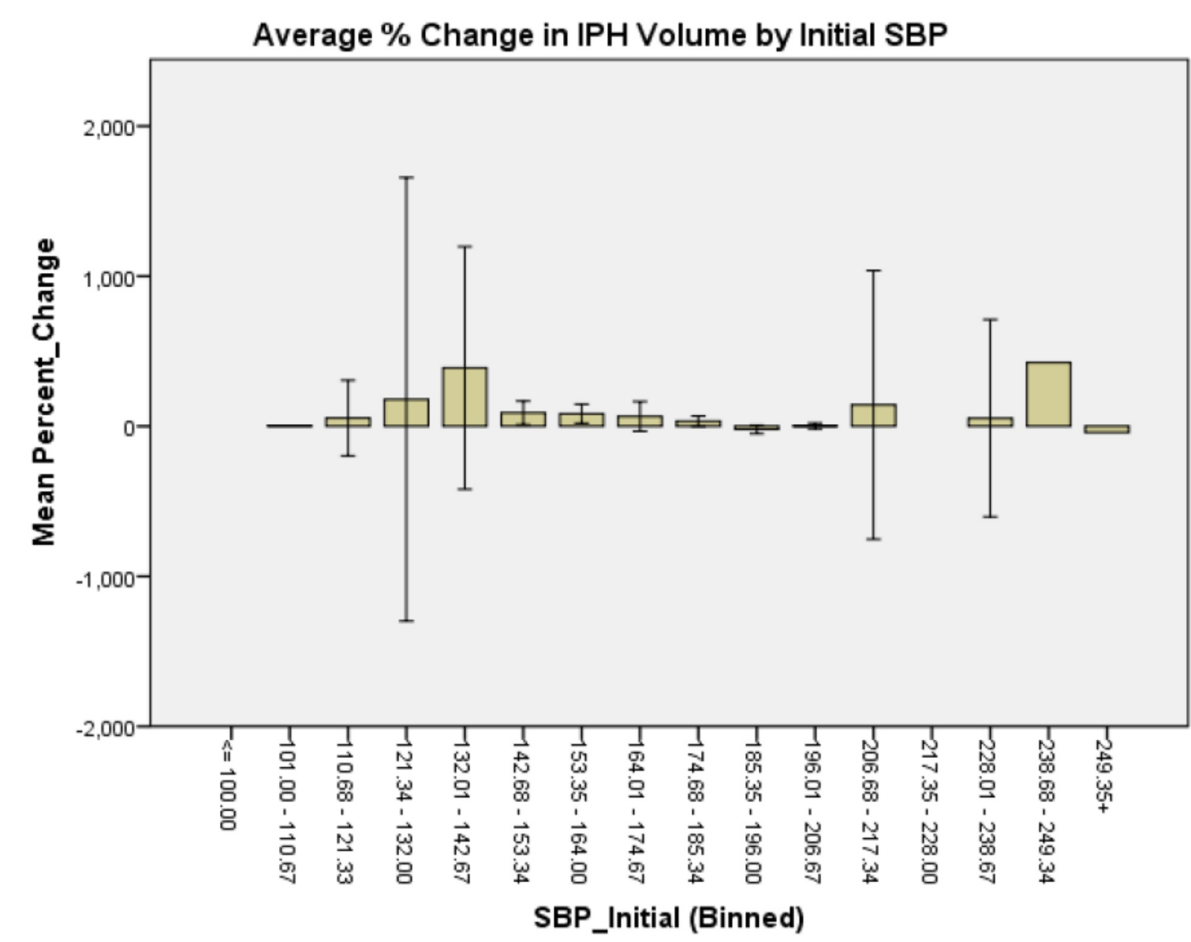

FIGURE 4: Average \% change in IPH volume by initial SBP.

There is a bimodal distribution of \% change in IPH size by initial SBP, with the largest expansion occurring in patients whose presenting SBP was 132-143 and those at 239-250.

Intraparenchymal hemorrhage: IPH

Systolic blood pressure: SBP

$\mathrm{SBP}(\mathrm{mmHg})$ at $1 \mathrm{hr}$

$101-110$

$111-121$

$122-132$

$133-142$

143-153

154-164

$165-175$

$>175$

\section{Mean \% IPH expansion}

411.5

34

45.1

268

51.1

60.9

$-4$

59.7

TABLE 2: Summary of mean percent IPH expansion by SBP range at one hour of presentation.

Systolic blood pressure: SBP

Intraparenchymal hemorrhage: IPH 


\section{Cureus}

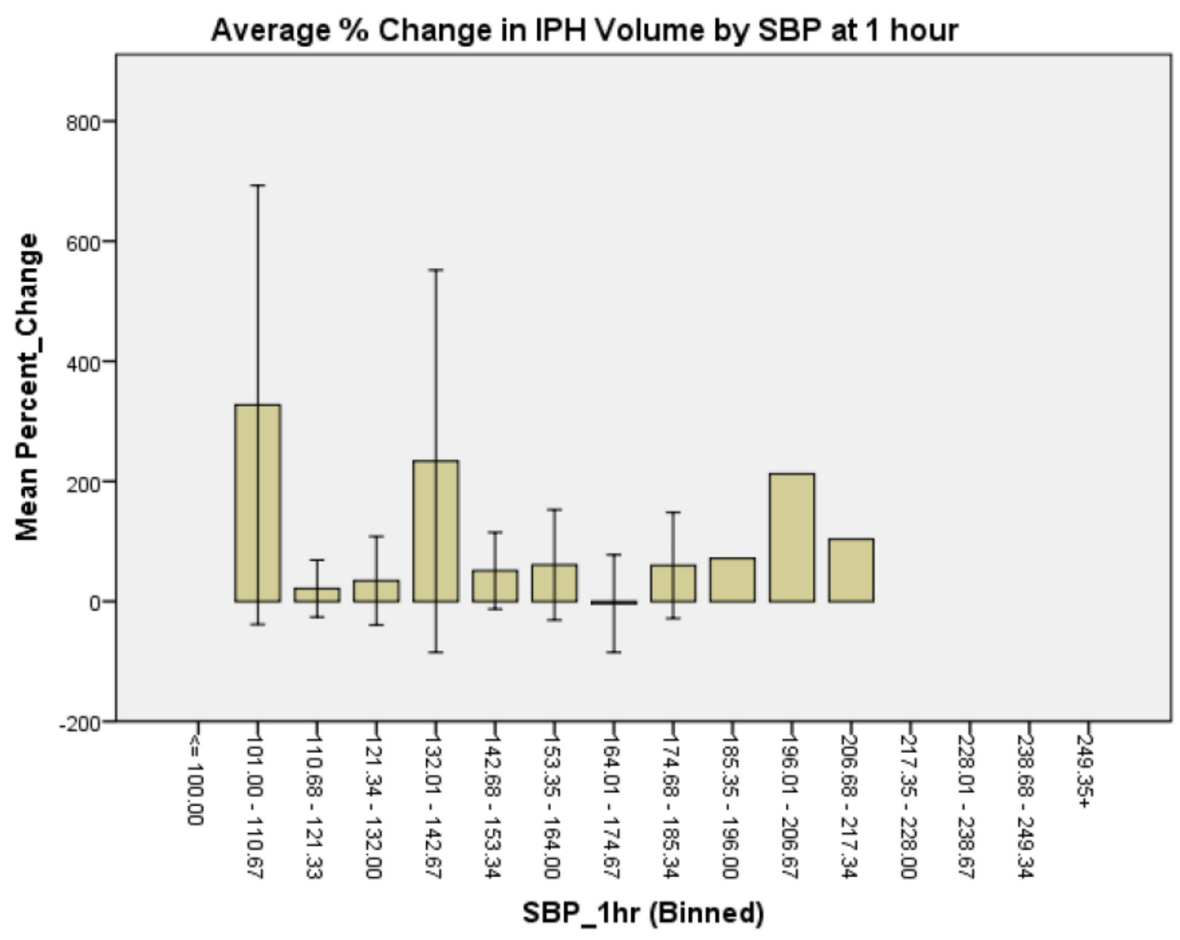

FIGURE 5: Average \% change in IPH volume by SBP at one hour of presentation.

Patients whose SBP fell between 132 and 143 again had larger IPH expansions. Of note, the largest IPH expansion occurred with SBP 101-111 and there was another relative maximum at 196-207.

Summary of Abbreviations in Figure 5:

Intraparenchymal Hemorrhage: IPH

Systolic Blood Pressure: SBP

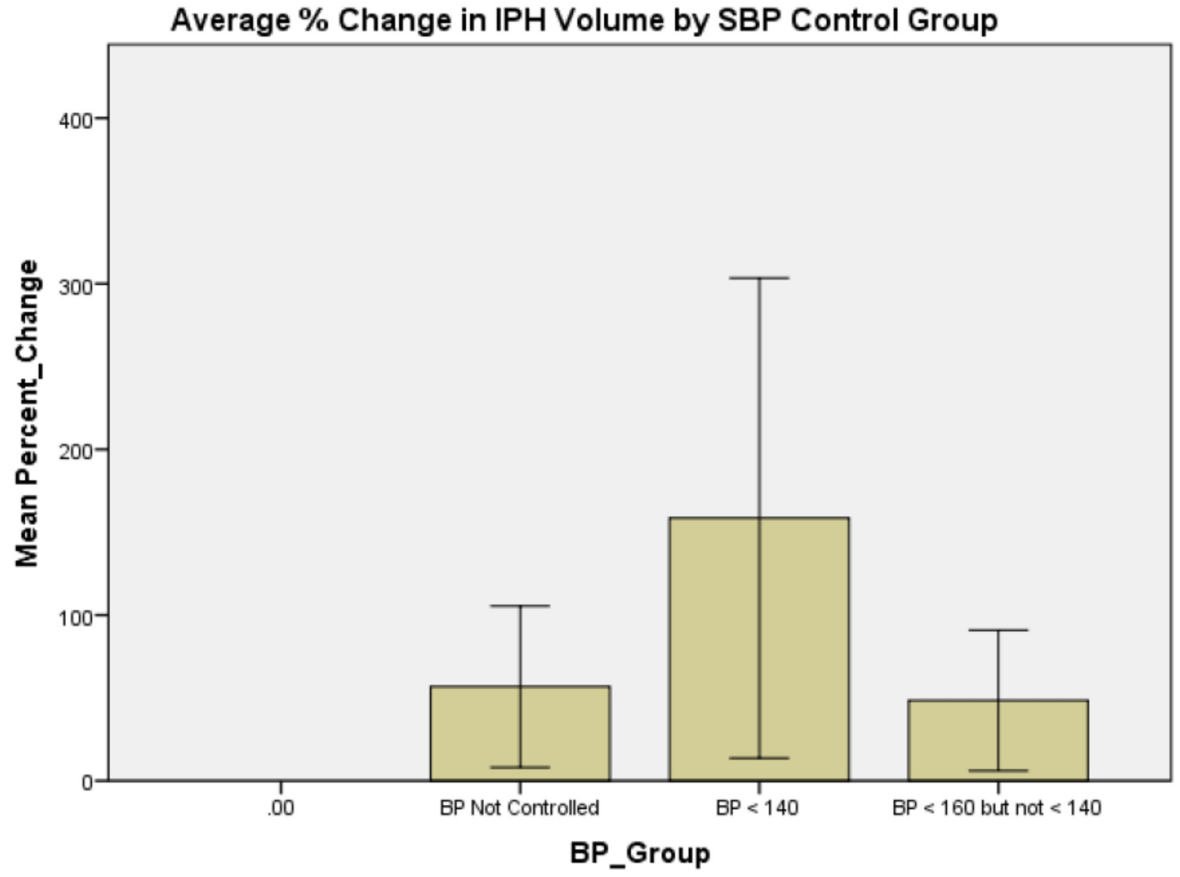

Error Bars: $95 \% \mathrm{Cl}$ 


\section{Cureus}

FIGURE 6: Average \% change in IPH volume by SBP control group.

Patients whose SBP was not controlled to $<160$ and patients whose SBP was controlled to $<160$ but not $<$ 140 appeared to have smaller IPH expansion than patients whose SBP was controlled to $<140$. There is, however, a large degree of variance in the data analyzed in this way.

Summary of Abbreviations in Figure 6:

Intraparenchymal Hemorrhage: IPH

Systolic Blood Pressure: SBP

Blood Pressure: BP

Factors Significantly Predicting Maximal IPH Expansion

\% Expansion of IPH

Patient Age

20 - 30 years

238.4

$60-70$ years

196.9

Initial IPH Volume

$65.23-78.26 \mathrm{ml}$

168.9

$0.01-13.04 \mathrm{ml}$

157.3

Presenting SBP

133-142 $\mathrm{mmHg}$

584.3

$>238 \mathrm{mmHg}$

761.8

$\mathrm{SBP}$ at $1 \mathrm{hr}$

$101-110 \mathrm{mmHg}$

411.5

$133-142 \mathrm{mmHg}$

268

\section{TABLE 3: Summary of factors that significantly affect IPH expansion, the two ranges of maximal} expansion, and the percent expansion relatable to that range.

Summary of Abbreviations in Table 3:

Intraparenchymal Hemorrhage: IPH

Systolic Blood Pressure: SBP

Factorial analysis revealed several significant two-way interactions. The interaction of age by BP group (as described above) was significant, $\chi 2(2)=231.962, p<0.0005$. The interaction of initial SBP with initial IPH volume was significant, $\chi 2(3)=171.443, p<0.0005$. The interaction of SBP at one hour of presentation and initial IPH volume was significant, $\chi 2(2)=288.190, p<0.0005$. Finally, the interaction of initial SBP and SBP at one hour of presentation was significant, $\chi 2(1)=121.677, \mathrm{p}<0.0005$.

\section{Discussion}

The data presented in this study exhibit several important findings. Patients in the 60-70 years age bracket tended to have the largest percent IPH expansion followed by the 20-30 years age group. This may be accounted for by the notion that patients age 20-30 years who present with IPH are likely to have inherent predisposition either epigenetically or by exogenous factors such as drug abuse to stroke. Moreover, patients in the 60-70 years group are known to be at high risk for stroke. Patients older than this group may, in fact, be likely to overcome intraparenchymal hemorrhage by inherent predispositions to vascular and parenchymal stability - and this may have conferred the survival advantage that has allowed them to reach advanced ages.

Initial volume of IPH on presentation also predicts IPH expansion with a group of patients who presented with initial IPH volume of 65.23 to $78.26 \mathrm{ml}$. There is also a second maximum in patients who presented with initial IPH volume of 0.01 to $13.04 \mathrm{ml}$. The expansion of the IPH in the group presenting initially with the smallest IPH is perhaps unsurprising as any expansion, even layering of blood, would be, in relative quantity, a large percentage change. Of more interest and clinical import, is the finding that patients who 
present with an initial bleed size between 65.23 and $78.26 \mathrm{ml}$ have predictably larger IPH expansion on follow-up imaging. These patients, therefore, may require closer monitoring and more stringent control of secondary factors such as coagulopathy, blood pressure, cholesterol, and cerebral perfusion pressure. Notably, patients whose initial IPH size was between $52.18 \mathrm{ml}$ and $65.22 \mathrm{ml}$ demonstrated a nadir in IPH expansion. This may perhaps represent the boundary of the brain parenchymal ability to compensate for hemorrhagic insult.

Taken in isolation, it would be easy to conclude that there exists a trend that patients whose SBP was controlled to $<140 \mathrm{mmHg}$ after IPH had larger IPH expansion than patients whose SBP was controlled to between 140 and $160 \mathrm{mmHg}$ (whose expansion was comparable to patients in whom SBP was not successfully controlled). A more nuanced approach, however, demonstrates that while patients did, in fact, have larger bleed expansion in the 132-143 mmHg managed group, patients whose SBP control fell lower than this bin, namely the 111-121 mmHg and 122-132 mmHg groups had relative minima in IPH expansion. This coincides with the AHA recommendation of ultimate SBP control in the face of IPH; however, this data suggest that if this control can reasonably be achieved early in the course of the disease, it may lead to smaller IPH expansion and, by extension, improved clinical outcomes. The relative maximum of bleed expansion in the 101-111 mmHg group may be attributable to derangements in CBF autoregulation that correspond to the low-normal SBP state experienced by these patients.

Future work in this arena may include patients with IPH from our other major centers to expand the sample size and to better approximate the population. Furthermore, future investigation may focus on patientspecific factors in future analyses such as comorbid conditions and confounding factors such as drug use and any potential coincident illnesses such as ventilator-associated pneumonia, cancers, etc. It is well studied that control of systolic blood pressure is critical in patients with intraparenchymal hemorrhage and tight control of blood pressure is a primary concern in the neurosurgical patient. A balance must be achieved wherein the systolic blood pressure is kept low enough to avoid injury and minimize bleeding while simultaneously being kept high enough to maintain perfusion, particularly in the setting of elevated intracranial pressure. Normal autoregulation of cerebral blood flow may be disturbed in pathologic states such as trauma, tumor, or hemorrhagic stroke, and therefore, exogenous control is often required in the neurosurgical patient. Current American Heart Association (AHA) guidelines recommend the maintenance of systolic blood pressure to less than $140 \mathrm{mmHg}$ in the setting of intracerebral hemorrhage with a longterm goal of SBP $<130 \mathrm{mmHg}$ [14]. Table 4 below summarizes several additional studies with blood pressure parameters, IPH expansion, and neurologic decline in their patient cohorts [2, 3, 10, 13, 14, 17, 18].

\begin{tabular}{|c|c|c|c|}
\hline Study & SBP target & $\%$ Hematoma expansion & $\%$ Early neurologic deterioration \\
\hline Koga et al. $(\mathrm{N}=211)[17]$ & $<160$ & 17.1 & $8.1 \%$ \\
\hline Li et al $(\mathrm{N}=87)[18]$ & $<140$ & $\begin{array}{l}21.9 \% \text { (Nicardipine), } 18.2 \% \\
\text { (Nimodipine) }\end{array}$ & $\begin{array}{l}\text { 28.3\% (Nicardipine), } 26.8 \% \\
\text { (Nimodipine) }\end{array}$ \\
\hline Hwang et al $(\mathrm{N}=88)$ [19] & $<140$ & $3.4 \%$ & $2.3 \%$ \\
\hline INTERACT ( $\mathrm{N}=404)$ [20] & $<140$ & $15 \%$ & $15 \%$ \\
\hline $\begin{array}{l}\text { INTERACT2 (N = 2794) } \\
\text { [21] }\end{array}$ & $<180$ & $26.1 \%$ & $17.9 \%$ \\
\hline ATACH $(\mathrm{N}=60)$ [22] & $\begin{array}{l}170-200 \text { vs } 140-170 \text { vs }< \\
140\end{array}$ & $33 \%$ vs $15 \%$ vs $32 \%$ & $5.5 \%$ vs $10 \%$ vs $18 \%$ \\
\hline ATACH2 $(\mathrm{N}=973)[23]$ & $<<140$ vs $<180$ & $18.9 \%$ vs $24.4 \%$ & $11 \%$ vs $8 \%$ \\
\hline
\end{tabular}

\section{TABLE 4: Summary of additional studies with regards to SBP targets and outcomes in patients with IPH}

Intraparenchymal hemorrhage: IPH

Systolic blood pressure: SBP

Intensive Blood Pressure Reduction in Acute Cerebral Hemorrhage Trial: INTERACT

Antihypertensive Treatment of Acute Cerebral Hemorrhage study: ATACH

As mentioned, careful balance is key. Increased intracranial pressure results in elevated interstitial hydrostatic pressure and compression of the cerebral vasculature. This results in decreased capillary flow 
hydrostatic gradient and increased cerebral vascular resistance. Further, this can lead to cerebral hypoxia and deficient metabolic reserve causing neuronal cell injury and death. Elevated mean arterial pressure (MAP) in the setting of hypertension leads to increased cerebral perfusion pressure (CPP) and can cause endothelial damage with the destruction of the blood-brain barrier and vasogenic edema leading to further intracranial hemorrhage.

\section{Conclusions}

Intraparenchymal hemorrhage is less common than ischemic stroke but has higher morbidity and mortality rates per incident. Therefore, urgent reduction in blood pressure can minimize hematoma expansion leading to a better outcome. Hematoma expansion can result in direct mass effect, cerebral edema, and intraventricular hemorrhage that leads to hydrocephalus. This leads to increased intracranial pressure and decreased cerebral perfusion pressure. At this time, nicardipine is the consensus intravenous continuous infusion drug of choice for emergent control of refractory hypertension; however, nimodipine, labetalol, esmolol, and other drips have been used with varying degrees of efficacy, complications, and clinical outcomes. The patient populations with the largest hematoma expansion are those who were 60-70 years and 20-30 years old and those whose initial ICH volume was 65.23 to $78.26 \mathrm{ml}$. While our data suggest that the ideal target range for SBP in the setting of acute IPH may be $121-132 \mathrm{mmHg}$, further evaluation of this patient population is required to definitively elucidate a target blood pressure range for the immediate postinsult period in intraparenchymal hemorrhage.

\section{Additional Information \\ Disclosures}

Human subjects: Consent was obtained by all participants in this study. Arrowhead Regional Medical Center Institutional Review Board issued approval 18-12. Animal subjects: All authors have confirmed that this study did not involve animal subjects or tissue. Conflicts of interest: In compliance with the ICMJE uniform disclosure form, all authors declare the following: Payment/services info: All authors have declared that no financial support was received from any organization for the submitted work. Financial relationships: All authors have declared that they have no financial relationships at present or within the previous three years with any organizations that might have an interest in the submitted work. Other relationships: All authors have declared that there are no other relationships or activities that could appear to have influenced the submitted work.

\section{References}

1. Qureshi AI, Ezzeddine MA, Nasar A, et al.: Prevalence of elevated blood pressure in 563,704 adult patients with stroke presenting to the ED in the United States. Am J Emerg Med. 2007, 25:32-38. 10.1016/j.ajem.2006.07.008

2. Tikhonoff V, Zhang H, Richart T, Staessen JA: Blood pressure as a prognostic factor after acute stroke . Lancet Neurol. 2009, 8:938-948. 10.1016/S1474-4422(09)70184-X

3. Ahmed N, Wahlgren N, Brainin M, et al.: Relationship of blood pressure, antihypertensive therapy, and outcome in ischemic stroke treated with intravenous thrombolysis: retrospective analysis from safe implementation of thrombolysis in stroke-international stroke thrombolysis register (SITS-ISTR). Stroke. 2009, 40:2442-2449. 10.1161/STROKEAHA.109.548602

4. Palm F, Henschke N, Wolf J, et. al: Intracerebral haemorrhage in a population-based stroke registry (LuSSt): Incidence, aetiology, functional outcome and mortality. J Neurol. 2013, 260:2541-2550. 10.1007/s00415013-7013-0

5. van Asch CJ, Luitse MJ, Rinkel GJ, van der Tweel I, Algra A, Klijn CJ: Incidence case fatality, and functional outcome of intracerebral haemorrhage over time, according to age, sex, and ethnic origin: a systematic review and meta-analysis. Lancet Neurol. 2010, 9:167-176. 10.1016/S1474-4422(09)70340-0

6. Guan J, Hawryluk GWJ: Targeting secondary hematoma expansion in spontaneous intracerebral hemorrhage - state of the art. Front Neurol. 2016, 7:187. 10.3389/fneur.2016.00187

7. Dandapani BK, Suzuki S, Kelley RE, Reyes-Iglesias Y, Duncan RC: Relation between blood pressure and outcome in intracerebral hemorrhage. Stroke. 1995, 26:21-24. 10.1161/01.STR.26.1.21

8. Wang X, Arima H, Al-Shahi Salman R, et al.: Rapid blood pressure lowering according to recovery at different time intervals after acute intracerebral hemorrhage: pooled analysis of the INTERACT studies. Cerebrovasc Dis. 2015, 39:242-248. 10.1159/000381107

9. Wang X, Arima H, Heeley E, et al.: Magnitude of blood pressure reduction and clinical outcomes in acute intracerebral hemorrhage: intensive blood pressure reduction in acute cerebral hemorrhage trial study. Hypertension. 2015, 65:1026-1032. 10.1161/HYPERTENSIONAHA.114.05044

10. Davis SM, Broderick J, Hennerici M, et al.: Hematoma growth is a determinant of mortality and poor outcome after intracerebral hemorrhage. Neurology. 2006, 66:1175-1181. 10.1212/01.wnl.0000208408.98482.99

11. Dowlatshahi D, Demchuk AM, Flaherty ML, Ali M, Lyden PL, Smith EE: Defining hematoma expansion in intracerebral hemorrhage: relationship with patient outcomes. Neurology. 2011, 76:1238-44. 10.1212/WNL.0b013e3182143317

12. Kazui S, Naritomi H, Yamamoto H, Sawada T, Yamaguchi T: Enlargement of spontaneous intracerebral hemorrhage: incidence and time course. Stroke. 1996, 27:1783-1787. 10.1161/01.STR.27.10.1783

13. Brott T, Broderick J, Kothari R, et al.: Early hemorrhage growth in patients with intracerebral hemorrhage . Stroke. 1997, 28:1-5. 10.1161/01.STR.28.1.1 
14. Hemphill JC, Greenberg SM, Anderson CS, et al.: Guidelines for the management of spontaneous intracerebral hemorrhage: a guideline for healthcare professionals from the American Heart Association/American Stroke Association. Stroke. 2015, 46:2032-2060. 10.1161/STR.0000000000000069

15. Sato S, Carcel C, Anderson CS: Blood pressure management after intracerebral hemorrhage. Curr Treat Options Neurol. 2015, 17:1-11. 10.1007/s11940-015-0382-1

16. Ko S-B, Choi HA, Parikh G, et al.: Multimodality monitoring for cerebral perfusion pressure optimization in comatose patients with intracerebral hemorrhage. Stroke. 2011, 42:3087-3092. 10.1161/STROKEAHA.111.623165

17. Koga M, Toyoda K, Yamagami H, et al.: Systolic blood pressure lowering to $160 \mathrm{mmHg}$ or less using nicardipine in acute intracerebral hemorrhage. J Hypertens. 2012, 30:2357-2364. 10.1097/HJH.0b013e328359311b

18. Li Y, Fang W, Tao L, et al.: Efficacy and safety of intravenous nimodipine administration for treatment of hypertension in patients with intracerebral hemorrhage. Neuropsychiatr Dis Treat. 2015, 11:1231-1238. 10.2147/NDT.S76882

19. Hwang S-K, Kim J-S, Kim JH, Hong CK, Yang KH: Antihypertensive treatment of acute intracerebral hemorrhage by intravenous nicardipine hydrochloride: prospective multi-center study. J Korean Med Sci. 2012, 27:1085-1090. 10.3346/jkms.2012.27.9.1085

20. Anderson CS, Huang Y, Wang JG, et al.: Intensive blood pressure reduction in acute cerebral haemorrhage trial (INTERACT): a randomised pilot trial. Lancet Neurol. 2008, 7:391-399. 10.1016/S1474-4422(08)70069-3

21. Anderson CS, Heeley E, Huang Y, et al.: Rapid blood-pressure lowering in patients with acute intracerebral hemorrhage. N Engl J Med. 2013, 368:2355-2365. 10.1056/NEJMoa1214609

22. Qureshi AI, Palesch YY, Martin R, et al.: Effect of systolic blood pressure reduction on hematoma expansion, perihematomal edema, and 3-month outcome among patients with intracerebral hemorrhage: results from the antihypertensive treatment of acute cerebral hemorrhage study. Arch Neurol. 2010, 67:570-576. 10.1001/archneurol.2010.61

23. Qureshi AI, Palesch YY, Barsan WG, et al.: Intensive blood-pressure lowering in patients with acute cerebral hemorrhage. N Engl J Med. 2016, 375:1033-1043. 10.1056/NEJMoa1603460 